\title{
Degrees of Coordination in Cognitive Networks
}

\author{
Holger Boche and Rafael F. Wyrembelski \\ Heinrich-Hertz-Lehrstuhl für Informationstheorie und theoretische Informationstechnik \\ Technische Universität Berlin, Berlin, Germany
}

\begin{abstract}
The paradigm shift from coordinated to uncoordinated wireless networks comes along with the need of new concepts for the frequency usage since interference will be an ubiquitous phenomenon. In this work, we analyze unknown varying interference in wireless networks with the help of the concept of arbitrarily varying channels. This concept shows that the unknown interference has a dramatic impact on the communication. In the case of high interference, it can happen that no communication is possible, not even at very low rates, if traditional interference coordination with pre-specified encoders and decoders is applied. We introduce more sophisticated coordination schemes as the transmitter-receiver or the correlated side information coordination. Here, an access to a common randomness is advantageously used to coordinate the choice of the encoders and decoders. This enables successfully a communication even in such situations where the traditional approach fails.
\end{abstract}

\section{Motivation}

The ongoing research progress reveals a paradigm shift from coordinated to uncoordinated wireless systems. While most current systems such as cellular systems are usually coordinated in a centralized way, several future systems will act in an uncoordinated and self-organizing way, e.g., ad-hoc or sensor networks. As an example Figure 1 depicts a wireless network which consists of several uncoordinated transmitterreceiver pairs or links, where each receiver receives the signal he is interested in but is also confronted with interfering signals from other transmitting nodes.

The main issue that comes along with this development is that interference becomes an ubiquitous phenomenon and will be one of the main impairments in future wireless networks. Since the resulting interference cannot be longer coordinated in a centralized way, new concepts are needed especially for the frequency usage.

Game theory seems to be a promising approach to model the behavior or the interaction of different transmitter-receiver pairs in such uncoordinated wireless networks. It allows to model strategies how the users act and provides a framework for the analysis of the performance of these strategies. Moreover, game theory is suitable to design (decentralized) algorithms. Obviously one is interested in strategies that require only small or no coordination between different transmitterreceiver links since for such strategies the needed overhead for the coordination will be small or negligible.

A survey of the application of game theory in the flat-fading two-user interference channel can be found in [1]. Transmit strategies for the MISO interference channel are discussed from a game-theoretic point of view in [2,3]. A general optimization framework is given in [4]. How game theory can

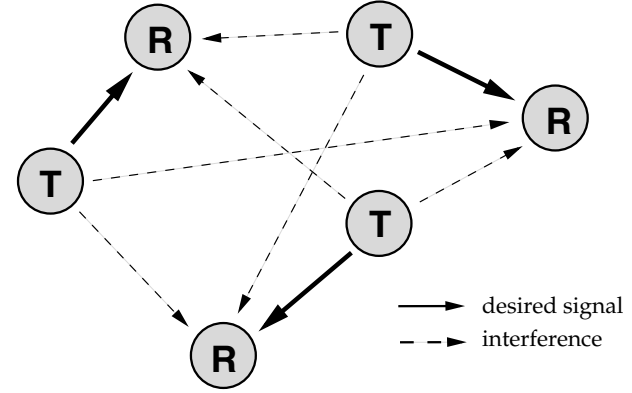

Fig. 1. Wireless network with several transmitter-receiver pairs. Each transmitter transmits a desired signal (solid) which simultaneously induces interference to all other receivers (dashed).

be used to design algorithms for resource allocation, medium access control, or power control can be found for example in [5-7] and in references therein. The concept of game theory is also successfully applied to cognitive radio scenarios, see for example $[8,9]$, where several users are allowed to use the same frequency resources. The social impact of optimal resource allocation in wireless networks is discussed in [10].

In this work, we analyze the impact of the interference in a wireless network with no coordination between different transmitter-receiver pairs. If there is no a priori knowledge about the strategies, e.g., the coding or modulation scheme, of the other transmitter-receiver links, there is no knowledge about the induced interference. The concept of arbitrarily varying channels (AVC) [11-14] provides a suitable and robust model for the treatment of the interference. In the following Section II we introduce the concept of the AVC and discuss the impact of the coordination within one transmitter-receiver link in Section III. An extension to a more complex multiuser setting is given in Section IV. Finally, we end up with a conclusion in Section V. ${ }^{1}$

\section{Modeling of Communication in Cognitive NETWORKS}

We start with the simplest interference scenario with two transmitter-receiver pairs (or links) where each receiver receives signals from both transmitters but is only interested in decoding the message from its own transmitter. We consider

\footnotetext{
${ }^{1}$ Notation: Discrete random variables are denoted by capital letters and their corresponding realizations and ranges by lower case letters and calligraphic letters respectively; $\mathbb{N}$ and $\mathbb{R}_{+}$denote the set of natural and non-negative real numbers; $\mathbb{E}[\cdot]$ is the expectation and $\mathcal{P}(\cdot)$ denotes the set of all probability distributions; $(\cdot)^{c}$ is the complement of a set; all logarithms, exponentials, and information quantities are taken to the basis 2 .
} 
the standard model with block codes of arbitrary but sufficient fixed length $n$. Let $\mathcal{M}_{k}:=\left\{1, \ldots, M_{k}^{(n)}\right\}, k=1,2$, be the set of messages to transmit.

Since in practical systems a transmitter usually uses a finite modulation scheme and a receiver quantizes the received signal before further processing, it is reasonable to assume finite input and output alphabets denoted by $\mathcal{X}_{k}$ and $\mathcal{Y}_{k}$ for link $k$, $k=1,2$, respectively. Then, for input and output sequences $x_{k}^{n} \in \mathcal{X}_{k}^{n}$ and $y_{k}^{n} \in \mathcal{Y}_{k}^{n}$ of length $n$ the transmission over the discrete memoryless channel is completely characterized by a stochastic matrix

$$
W_{k}^{\otimes n}\left(y_{k}^{n} \mid x_{1}^{n}, x_{2}^{n}\right):=\prod_{i=1}^{n} W_{k}\left(y_{k, i} \mid x_{1, i}, x_{2, i}\right), \quad k=1,2 .
$$

Thereby, the additive noise at the receivers is taken into account by considering stochastic matrices and not deterministic ones. Interestingly, the transmission model in (1) looks like a multiple access channel, since the received signal depends both on the intended message and the interfering message from the other link.

Remark 1: If one considers the received signal from the other transmitter as additional noise, one would end up with a modified stochastic matrix $\tilde{W}_{k}^{\otimes n}\left(y_{k}^{n} \mid x_{k}^{n}\right), k=1,2$, where the received signal depends only on the intended message.

The coding strategy for transmitter-receiver pair $k, k=1,2$, is specified by the following definition.

Definition 1: A $\left(M_{k}^{(n)}, n\right)$-code or codebook $\mathcal{C}_{k}$ is a family

$$
\mathcal{C}_{k}:=\left\{\left(x_{m_{k}}^{n}, D_{m_{k}}^{(k)}\right): m_{k} \in \mathcal{M}_{k}\right\}
$$

with codewords $x_{m_{k}}^{n} \in \mathcal{X}_{k}^{n}$, one for each message $m_{k} \in$ $\mathcal{M}_{k}$, and decoding sets $D_{m_{k}}^{(k)} \subseteq \mathcal{Y}_{k}^{n}$ for all $m_{k} \in \mathcal{M}_{k}$ with $D_{m_{k}}^{(k)} \cap D_{m_{k}^{\prime}}^{(k)}=\emptyset$ for $m_{k} \neq m_{k}^{\prime}$.

When $x_{m_{1}}^{n}$ and $x_{m_{2}}^{n}$ have been sent according to fixed codebooks $\mathcal{C}_{1}$ and $\mathcal{C}_{2}$, and $y_{1}^{n}$ and $y_{2}^{n}$ have been received, the decoder of receiver $k$ is in error if $y_{k}^{n} \notin D_{m_{k}}^{(k)}, k=1,2$. With this, we can define the probability of error at receiver $k$ for given messages $m_{1}$ and $m_{2}$ as

$$
e_{k}\left(m_{1}, x_{m_{2}}^{n}\right):=W_{k}^{\otimes n}\left(\left(D_{m_{k}}^{(k)}\right)^{c} \mid x_{m_{1}}^{n}, x_{m_{2}}^{n}\right)
$$

and the average probability of error at node 1 as

$$
\bar{e}_{1}\left(x_{m_{2}}^{n}\right):=\frac{1}{M_{1}^{(n)}} \sum_{m_{1}=1}^{M_{1}^{(n)}} e_{1}\left(m_{1}, x_{m_{2}}^{n}\right)
$$

with a similar expression for node 2 .

Important to note is that the probability of error depends on the codebooks both transmitter-receiver pairs use as well as on the specific message the interfering transmitter sends.

Definition 2: A rate $R_{k} \in \mathbb{R}_{+}$is said to be achievable if for any $\delta>0$ there exists an $n(\delta) \in \mathbb{N}$ and a sequence of $\left(M_{k}^{(n)}, n\right)$-codes, $k=1,2$, such that for all $n \geq n(\delta)$ we have

$$
\frac{\log M_{k}^{(n)}}{n} \geq R_{k}-\delta
$$

while $\max _{x_{m_{2}}^{n}} \bar{e}_{1}\left(x_{m_{2}}^{n}\right), \max _{x_{m_{1}}^{n}} \bar{e}_{2}\left(x_{m_{1}}^{n}\right) \rightarrow 0$ as $n \rightarrow \infty$.
If we assume no coordination between both transmitterreceiver pairs, there is no a priori knowledge about the used codebooks and the codewords that are chosen by the interfering transmitter. Consequently, the receiver can be confronted with arbitrary interfering sequences. This corresponds to the concept of arbitrarily varying channels (AVC) [11-14] and the only way to guarantee a successful transmission is to find a universal strategy that works for all possible codebooks and interfering codewords simultaneously.

To model the appearance of arbitrary interfering sequences, we introduce a finite state set $\mathcal{S}$. Then, for a fixed state sequence $s^{n} \in \mathcal{S}^{n}$ of length $n$ and input and output sequences $x^{n} \in \mathcal{X}^{n}$ and $y^{n} \in \mathcal{Y}^{n}$, the discrete memoryless channel is given by $W^{\otimes n}\left(y^{n} \mid x^{n}, s^{n}\right):=\prod_{i=1}^{n} W\left(y_{i} \mid x_{i}, s_{i}\right) .^{2}$

Definition 3: The discrete memoryless arbitrarily varying channel (AVC) $\mathcal{W}$ is the family

$$
\mathcal{W}:=\left\{W^{\otimes n}: \mathcal{X}^{n} \times \mathcal{S}^{n} \rightarrow \mathcal{P}\left(\mathcal{Y}^{n}\right)\right\}_{n \in \mathbb{N}, s^{n} \in \mathcal{S}^{n}} .
$$

Further, for any probability distribution $q \in \mathcal{P}(\mathcal{S})$ we denote the averaged channel by $\bar{W}^{(q)}(y \mid x):=\sum_{s \in \mathcal{S}} W(y \mid x, s) q(s)$.

We denote the mutual information between the input random variable $X$ and the output random variable $Y$ by $I(X ; Y)$ [13, p. 21]. Moreover, to emphasize the dependency of the mutual information on the input distribution $p \in \mathcal{P}(\mathcal{X})$ and the channel $W$, we also write $I(X ; Y)=I(p, W)$ interchangeably.

\section{TYPES OF COORDINATION IN ONE TRANSMITTER-RECEIVER PAIR}

In the following we analyze different approaches of coordination in one transmitter-receiver pair and specify their impact on the transmission. Therefore, we characterize all achievable rates at which reliable communication is possible for three different types of coordination, namely the traditional interference, the transmitter-receiver, and the correlated side information coordination.

\section{A. Traditional Interference Coordination}

The system design of the traditional or conventional interference coordination is defined by a deterministic strategy, where the transmitter and the receiver use a pre-specified encoder and decoder as given in Definition 1. To state the following result, we need the concept of symmetrizability.

Definition 4: An AVC is symmetrizable if for some channel $\varphi: \mathcal{X} \rightarrow \mathcal{P}(\mathcal{S})$

$$
\sum_{s \in \mathcal{S}} W(y \mid x, s) \varphi\left(s \mid x^{\prime}\right)=\sum_{s \in \mathcal{S}} W\left(y \mid x^{\prime}, s\right) \varphi(s \mid x)
$$

holds for every $x, x^{\prime} \in \mathcal{X}$ and $y \in \mathcal{Y}$.

For the traditional interference coordination the capacity is known [12-14] and summarized in the following theorem.

\footnotetext{
${ }^{2}$ In the following we drop the subscript $(\cdot)_{k}$ indicating the transmitterreceiver pair, since obviously the argumentation holds for all $k$.
} 
Theorem 1: For a non-symmetrizable $\operatorname{AVC} \mathcal{W}$ the capacity $C$ under the average error criterion is

$$
C=\max _{p \in \mathcal{P}(\mathcal{X})} \inf _{q \in \mathcal{P}(\mathcal{S})} I\left(p, \bar{W}^{(q)}\right) .
$$

For a symmetrizable AVC the capacity is zero, i.e., $C=0$.

The complete proof can be found in [14]. In the following we only want to highlight the key insight why we have a zero capacity if the AVC is symmetrizable.

Let $x_{m}^{n} \in \mathcal{X}^{n}, m=1, \ldots, M^{(n)}$ with $M^{(n)} \geq 2$ be arbitrary codewords. For a symmetrizable AVC, we can consider interfering sequences that look like valid codewords, more precisely $s_{m}^{n}=x_{m}^{n}, m=1, \ldots, M^{(n)}$. Now, for each pair of codewords $(k, l) \in \mathcal{M} \times \mathcal{M}$ with $k \neq l$ we have for the probability of error

$$
\begin{aligned}
& \mathbb{E}\left[e\left(k, s_{l}^{n}\right)\right]+\mathbb{E}\left[e\left(l, s_{k}^{n}\right)\right] \\
= & \mathbb{E}\left[W^{\otimes n}\left(\left(D_{k}\right)^{c} \mid x_{k}^{n}, s_{l}^{n}\right)\right]+\mathbb{E}\left[W^{\otimes n}\left(\left(D_{l}\right)^{c} \mid x_{l}^{n}, s_{k}^{n}\right)\right] \\
= & \mathbb{E}\left[W^{\otimes n}\left(\left(D_{k}\right)^{c} \mid x_{k}^{n}, s_{l}^{n}\right)\right]+\mathbb{E}\left[W^{\otimes n}\left(\left(D_{l}\right)^{c} \mid x_{k}^{n}, s_{l}^{n}\right)\right] \\
\geq & \mathbb{E}\left[W^{\otimes n}\left(\left(D_{k}\right)^{c} \mid x_{k}^{n}, s_{l}^{n}\right)\right]+\mathbb{E}\left[W^{\otimes n}\left(D_{k} \mid x_{k}^{n}, s_{l}^{n}\right)\right] \\
= & \mathbb{E}\left[W^{\otimes n}\left(\left(D_{k}\right)^{c} \cup D_{k} \mid x_{k}^{n}, s_{l}^{n}\right)\right]=1
\end{aligned}
$$

where the second equality follows from the fact that the AVC is symmetrizable, cf. Definition 4. Hence, this leads for the average probability of error to

$$
\begin{aligned}
\frac{1}{M^{(n)}} \sum_{l=1}^{M^{(n)}} \mathbb{E}\left[\bar{e}\left(s_{l}^{n}\right)\right] & =\frac{1}{\left(M^{(n)}\right)^{2}} \sum_{k=1}^{M^{(n)}} \sum_{l=1}^{M^{(n)}} \mathbb{E}\left[e\left(k, s_{l}^{n}\right)\right] \\
& \geq \frac{1}{\left(M^{(n)}\right)^{2}} \cdot \frac{M^{(n)}\left(M^{(n)}-1\right)}{2} \\
& =\frac{M^{(n)}-1}{2 M^{(n)}} \geq \frac{1}{4}
\end{aligned}
$$

which implies that $\mathbb{E}\left[\bar{e}\left(s_{l}^{n}\right)\right] \geq \frac{1}{4}$ for at least one $l \in \mathcal{M}$. Since the average probability of error is bounded from below by a positive constant, a reliable transmission is not possible so that we have $C=0$ if the AVC is symmetrizable.

This becomes intuitively clear, if one realizes the following. Since the AVC is symmetrizable, cf. (2), it can happen that the interfering sequence looks like another valid codeword. Then, the receiver receives a superimposed version of two valid codewords and cannot distinguish anymore which of the codewords was transmitted by the transmitter and which one is the interference so that reliable communication can no longer be guaranteed.

\section{B. Transmitter-Receiver Coordination}

Since the traditional interference coordination with predetermined encoder and decoder fails in the case of symmetrizable channels, we are interested in strategies that work well also in this case. Therefore, we consider in the following a strategy with a more involved coordination, where we allow the transmitter and the receiver to coordinate their choice of the encoder and decoder based on an access to a common resource which is independent of the current message. This leads directly to the following definition.
Definition 5: A random $\left(M^{(n)}, n, \mathcal{U}, \mu\right)$-code $\mathcal{C}_{\mathrm{cr}}$ for the AVC is a collection of deterministic $\left(M^{(n)}, n\right)$-codes $\mathcal{C}(U)$, $U \in \mathcal{U}, U \sim \mu$, and

$$
\mathcal{C}(U):=\left\{\left(x_{m}^{n}(U), D_{m}(U)\right): m \in \mathcal{M}\right\}
$$

where $\mu$ is the uniform distribution on $\mathcal{U}$.

This means that the codewords and the decoding sets are chosen according to a common random experiment, realized in Definition 5 by the random variable $U$, whose outcome has to be known to the transmitter and the receiver in advance. Clearly, the definitions of probability of error, a randomly achievable rate, and the random code capacity $C_{\mathrm{cr}}$ follow accordingly.

The access to the common random variable can be realized by an external source, e.g., a satellite signal. Moreover, we know from [12] that if we transmit at rate $R$ with exponentially many messages, i.e., $\exp (n R)$, it suffices to use a random code which consists of $n^{2}$ encoder-decoder pairs and a uniformly distributed random variable whose value indicates which of the pair the transmitter and the receiver have to use.

Due to the additional degree of coordination within one transmitter-receiver pair we expect an improvement in the performance compared to the traditional coordination approach especially for the case of symmetrizable channels. The following result confirms our intuition $[11,13]$.

Theorem 2: The random code capacity $C_{\mathrm{cr}}$ of the $\mathrm{AVC}$ is

$$
C_{\mathrm{cr}}=\max _{p \in \mathcal{P}(\mathcal{X})} \inf _{q \in \mathcal{P}(\mathcal{S})} I\left(p, \bar{W}^{(q)}\right) .
$$

It shows that the random code capacity $C_{\text {cr }}$ has the same value as for the traditional interference coordination but is also achieved in the case of symmetrizable channels.

\section{Correlated Side Information}

For the transmitter-receiver coordination we assumed that both, the transmitter and the receiver, have an access to a common random experiment. This seems to be a hard condition and one can think of a weaker version. Therefore, we allow in this section the transmitter and the receiver each to have access to an own random experiment which are both correlated. In more detail, the correlated side information strategy is given by the following definition.

Definition 6: A correlated $\left(M^{(n)}, n, \mathcal{U}, \mathcal{V}, \mu, \nu\right)$-code $C_{\text {corr }}$ for the AVC is a family of deterministic $\left(M^{(n)}, n\right)$-codes $\mathcal{C}\left(U^{n}, V^{n}\right), U^{n} \in \mathcal{U}^{n}, V^{n} \in \mathcal{V}^{n}, U \sim \mu, V \sim \nu$, and

$$
\mathcal{C}\left(U^{n}, V^{n}\right):=\left\{\left(x_{m}^{n}\left(U^{n}\right), D_{m}\left(V^{n}\right)\right): m \in \mathcal{M}\right\}
$$

with $I(U ; V)>0$ and $\mu, \nu$ are the uniform distributions on $\mathcal{U}$ and $\mathcal{V}$ respectively.

Thereby, the fact that the random variables $U$ and $V$ are correlated, is guaranteed by the weak condition $I(U ; V)>0$. Note that in contrast to the transmitter-receiver coordination, the codewords and decoding sets depend now on a whole sequence of the random variables.

The next result states the capacity for the case of correlated side information at the transmitter and the receiver [15]. 
Theorem 3: The correlated side information capacity $C_{\mathrm{corr}}$ of the AVC is

$$
C_{\text {corr }}=\max _{p \in \mathcal{P}(\mathcal{X})} \inf _{q \in \mathcal{P}(\mathcal{S})} I\left(p, \bar{W}^{(q)}\right) .
$$

The theorem shows that even if the transmitter and the receiver only have access to correlated versions of a random experiment, this side information is already sufficient to achieve the same rates as for the transmitter-receiver coordination. It shows that correlated side information suffices to overcome symmetrizable channel conditions.

\section{Gaussian AVC}

Here, we show that the results derived so far for discrete channels also extend to the Gaussian AVC where the flatfading input-output relation for one time instant between the transmitter and the receiver is given by

$$
y=x+i+n
$$

with $y \in \mathbb{R}$ the output, $x \in \mathbb{R}$ the input, $i \in \mathbb{R}$ the additive interference, and $n \sim \mathcal{N}\left(0, \sigma^{2}\right)$ the additive Gaussian noise. Similar to the discrete AVC we are in a comfortable situation since the Gaussian AVC is also well studied in [16, 17].

We assume that the transmitter and the interferer are restricted by average power constraints $\Gamma$ and $\Lambda$, respectively. This means, all permissible input sequences $x^{n}=$ $\left(x_{1}, x_{2}, \ldots, x_{n}\right)$ of length $n$ are those who satisfy

$$
\left\|x^{n}\right\|^{2}=\sum_{j=1}^{n} x_{j}^{2} \leq n \Gamma
$$

and, accordingly, all permissible interfering sequences $i^{n}=$ $\left(i_{1}, i_{2}, \ldots, i_{n}\right)$ of length $n$ are those who satisfy

$$
\left\|i^{n}\right\|^{2}=\sum_{j=1}^{n} i_{j}^{2} \leq n \Lambda .
$$

From conditions (3) and (4) follow that all permissible codewords and interfering sequences lie on or within an $n$ dimensional sphere of radius $\sqrt{n \Gamma}$ or $\sqrt{n \Lambda}$, respectively.

For the traditional interference coordination we end up with the following result [14].

Theorem 4: The capacity $C$ of the Gaussian AVC with input constraint $\Gamma$ and interfering constraint $\Lambda$ is

$$
C= \begin{cases}\frac{1}{2} \log \left(1+\frac{\Gamma}{\Lambda+\sigma^{2}}\right), & \text { if } \Gamma>\Lambda \\ 0, & \text { if } \Gamma \leq \Lambda\end{cases}
$$

Remark 2: Interestingly, the result shows that the existence of positive rates only depends on the interference and not on the noise. This manifests the intuition that the goal of the traditional interference coordination is to ensure that the received interference will be small enough.

Remark 3: Important to note is that for high interference there is no reliable communication possible, not even at very low rates. Consequently, for the traditional interference coordination the well-known log expression is only valid for the case of low interference.

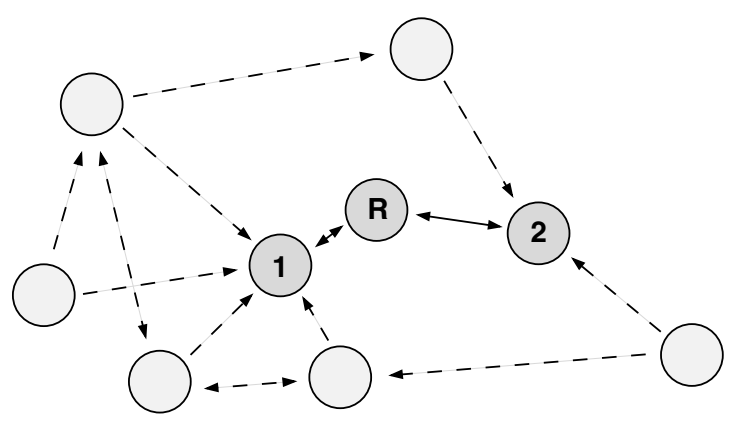

Fig. 2. Bidirectional relaying in a wireless network.

The transmitter-receiver coordination is studied in [16] and yields the following.

Theorem 5: The random code capacity $C_{\mathrm{cr}}$ of the Gaussian AVC with input constraint $\Gamma$ and interfering constraint $\Lambda$ is

$$
C_{\mathrm{cr}}=\frac{1}{2} \log \left(1+\frac{\Gamma}{\Lambda+\sigma^{2}}\right) .
$$

Remark 4: The random code capacity $C_{\mathrm{cr}}$ is identical to the one if the interfering sequences would consist of iid Gaussian symbols distributed according to $\mathcal{N}(0, \Lambda)$. This means, the arbitrary, possibly non-Gaussian, unknown interference do not more affect the achievable rate than Gaussian noise of the same power.

Remark 5: We presented the results for transmitter-receiver pairs where each one is equipped with a single antenna only. There is some work on the vector-valued case as well but it is out of scope of this work. The random code capacity of the vector-valued Gaussian AVC is given in [18]. Some work on the deterministic counterpart can be found in [19].

\section{CoOrdination in a More Complicated Network}

So far, we considered a single link in the presence of arbitrarily varying interference. Here, we give an example that the presented techniques also extend to a more complex multiuser setting.

In this section, we consider bidirectional relaying where a half-duplex relay node establishes a bidirectional communication between two other nodes using a decode-and-forward protocol [20-23]. This is also known as two-way relaying. In the initial multiple access phase, both nodes transmit their messages to the relay node which decodes them. In the succeeding bidirectional broadcast phase, the relay broadcasts a re-encoded composition of them which allows both nodes to decode the other's message using their own message from the previous phase as side information.

We assume that the communication is disturbed by additive arbitrarily varying interference as depicted in Figure 2. Thereby, we concentrate on the bidirectional broadcast phase, which is called the arbitrarily varying bidirectional broadcast channel (AVBBC). For an AVBBC the input-output relation between the relay node and node $k$ is given by

$$
y_{k}=x+i_{k}+n_{k}, \quad k=1,2 .
$$


Since the interference at both receivers may differ, we consider two interfering constraints $\Lambda_{1}$ and $\Lambda_{2}$, one for each receiver, in a similar way as in done in Section III-D for a single transmitter-receiver pair.

Using the similar ideas and techniques as for the Gaussian AVC in Section III-D we are able to derive similar results for the AVBBC. The traditional interference coordination leads to the following capacity region [24].

Theorem 6: The capacity region of the AVBBC with input constraint $\Gamma$ and interfering constraints $\Lambda_{1}$ and $\Lambda_{2}$ is the set of all rate pairs $\left(R_{\mathrm{R} 1}, R_{\mathrm{R} 2}\right) \in \mathbb{R}_{+}^{2}$ satisfying

$$
R_{\mathrm{R} k} \leq \begin{cases}\frac{1}{2} \log \left(1+\frac{\Gamma}{\Lambda_{k}+\sigma^{2}}\right) & \text { if } \Gamma>\Lambda_{k} \\ 0 & \text { if } \Gamma \leq \Lambda_{k}\end{cases}
$$

$k=1,2$, where $R_{\mathrm{R} k}$ denotes the rate between the relay and node $k$. This means the interior of the capacity region is nonempty if and only if $\Gamma>\Lambda_{1}$ and $\Gamma>\Lambda_{2}$.

The theorem shows that similar to the Gaussian AVC, there is no reliable communication possible if the power of the interference exceeds the transmit power. Since we have two interfering constraints, for a reliable bidirectional communication we have to ensure that both interfering constraints are small enough. Further, for the more sophisticated transmitterreceiver coordination we obtain the following [24].

Theorem 7: The random code capacity region of the AVBBC with input constraint $\Gamma$ and interfering constraints $\Lambda_{1}$ and $\Lambda_{2}$ is the set of all rate pairs $\left(R_{\mathrm{R} 1}, R_{\mathrm{R} 2}\right) \in \mathbb{R}_{+}^{2}$ satisfying

$$
R_{\mathrm{R} k} \leq \frac{1}{2} \log \left(1+\frac{\Gamma}{\Lambda_{k}+\sigma^{2}}\right), \quad k=1,2 .
$$

Again, a more sophisticated coordination enables also in this multi-user setting an improvement in the performance. The coordination allows to transmit successfully even in the case of high interference at both receiving nodes.

\section{CONCLUSION}

We see that unknown varying interference has a dramatic impact on the communication in a wireless system. If the traditional interference coordination is applied, the unknown varying interference can lead to situations which completely prohibit any reliable communication. This is mainly based on the assumption that the traditional coordination treats the interference as some kind of additional noise. As we have seen, this is in general to imprecise and leads to a performance loss especially if the interference is caused by other transmitters that use the same or a similar codebook. Consequently, a traditional interference coordination is only reasonable if the interference can be made small enough.

It shows that a more sophisticated coordination within one transmitter-receiver link as the transmitter-receiver coordination or the correlated side information coordination can overcome this problem. Therewith, it is possible to transmit reliably even in such situations.

\section{REFERENCES}

[1] E. Larsson, E. A. Jorswieck, J. Lindblom, and R. Mochaourab, "Game Theory and the Flat-Fading Gaussian Interference Channel," IEEE Signal Process. Mag., vol. 26, no. 5, pp. 18-27, Sep. 2009.

[2] E. A. Jorswieck and E. Larsson, "Complete Characterization of the Pareto Boundary for the MISO Interference Channel," IEEE Trans. Signal Process., vol. 56, no. 10, pp. 5292-5296, Oct. 2008.

[3] J. Lindblom, E. A. Jorswieck, and E. Larsson, "Parameterization of the MISO IFC Rate Region: The Case of Partial Channel State Information," IEEE Trans. Wireless Commun., vol. 9, no. 2, pp. 500-504, Feb. 2010.

[4] E. A. Jorswieck and E. Larsson, "Monotonic Optimization Framework for the MISO Interference Channel," IEEE Trans. Commun., 2010, will appear.

[5] F. Meshkati, M. Chiang, H. V. Poor, and S. C. Schwartz, "A GameTheoretic Approach to Energy-Efficient Power Control in Multicarrier CDMA Systems," IEEE J. Sel. Areas Commun., vol. 24, no. 6, pp. 11151129, Jun. 2006.

[6] F. Meshkati, H. V. Poor, and S. C. Schwartz, "Energy-Efficient Resource Allocation in Wireless Networks," IEEE Signal Process. Mag., vol. 24, no. 3, pp. 58-68, May 2007.

[7] S. M. Betz and H. V. Poor, "Energy Efficient Communications in CDMA Networks: A Game Theoretic Analysis Considering Operating Costs," IEEE Trans. Signal Process., vol. 56, no. 10, pp. 5181-5190, Oct. 2008.

[8] D. P. Palomar, J. M. Cioffi, and M. A. Lagunas, "Uniform Power Allocation in MIMO Channels: A Game-Theoretic Approach," IEEE Trans. Inf. Theory, vol. 49, no. 7, pp. 1707-1727, Jul. 2003.

[9] G. Scutari and D. P. Palomar, "MIMO Cognitive Radio: A Game Theoretical Approach," IEEE Trans. Signal Process., vol. 58, no. 2, pp. 761-780, Feb. 2010.

[10] H. Boche, S. Naik, and T. Alpcan, "Characterization of NonManipulable and Pareto Optimal Resource Allocation Strategies for Interference Coupled Wireless Systems," in Proc. IEEE Conf. on Computer Commun., San Diego, USA, Mar. 2010.

[11] D. Blackwell, L. Breiman, and A. J. Thomasian, "The Capacities of Certain Channel Classes under Random Coding," Ann. Math. Stat., vol. 31, no. 3, pp. 558-567, 1960.

[12] R. Ahlswede, "Elimination of Correlation in Random Codes for Arbitrarily Varying Channels," Z. Wahrscheinlichkeitstheorie verw. Gebiete, vol. 44, pp. 159-175, 1978.

[13] I. Csiszár and J. Körner, Information Theory - Coding Theorems for Discrete Memoryless Systems. Academic Press, 1981.

[14] I. Csiszár and P. Narayan, "The Capacity of the Arbitrarily Varying Channel Revisited: Positivity, Constraints," IEEE Trans. Inf. Theory, vol. 34, no. 2, pp. 181-193, Mar. 1988.

[15] R. Ahlswede and N. Cai, "Correlated sources help the transmission over AVC," IEEE Trans. Inf. Theory, vol. 43, no. 4, pp. 1254-1255, Jul. 1997.

[16] B. Hughes and P. Narayan, "Gaussian Arbitrarily Varying Channels," IEEE Trans. Inf. Theory, vol. 33, no. 2, pp. 267-284, Mar. 1987.

[17] I. Csiszár and P. Narayan, "Capacity of the Gaussian Arbitrarily Varying Channel," IEEE Trans. Inf. Theory, vol. 37, no. 1, pp. 18-26, Jan. 1991.

[18] B. Hughes and P. Narayan, "The Capacity of a Vector Gaussian Arbitrarily Varying Channel," IEEE Trans. Inf. Theory, vol. 34, no. 5, pp. 995-1003, Sep. 1988

[19] I. Csiszár, "Arbitrarily Varying Channels with General Alphabets and States," IEEE Trans. Inf. Theory, vol. 38, no. 6, pp. 1725-1742, Nov. 1992.

[20] B. Rankov and A. Wittneben, "Spectral Efficient Protocols for HalfDuplex Fading Relay Channels," IEEE J. Sel. Areas Commun., vol. 25, no. 2, pp. 379-389, Feb. 2007.

[21] P. Larsson, N. Johansson, and K.-E. Sunell, "Coded Bi-directional Relaying," in Proc. 5th Scandinavian Workshop on Ad Hoc Networks, Stockholm, Sweden, May 2005, pp. 851-855.

[22] T. J. Oechtering, C. Schnurr, I. Bjelaković, and H. Boche, "Broadcast Capacity Region of Two-Phase Bidirectional Relaying," IEEE Trans. Inf. Theory, vol. 54, no. 1, pp. 454-458, Jan. 2008.

[23] S. J. Kim, P. Mitran, and V. Tarokh, "Performance Bounds for Bidirectional Coded Cooperation Protocols," IEEE Trans. Inf. Theory, vol. 54, no. 11, pp. 5235-5241, Nov. 2008.

[24] R. F. Wyrembelski, I. Bjelaković, and H. Boche, "Bidirectional Relaying in Wireless Networks - Impact of Degree of Coordination," in Proc. IEEE Int. Conf. Acoustics, Speech, and Signal Process., Dallas, TX, USA, Mar. 2010. 